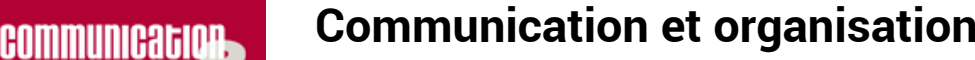

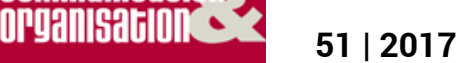

Les nouvelles cultures de l'information dans les organisations

\section{Analyse du discours des acteurs en charge de la lutte contre la criminalité financière}

An analysis of the discourse of the players in charge of the fight against financial criminality

\section{Frédéric Compin}

\section{OpenEdition}

\section{Journals}

Édition électronique

URL : http://journals.openedition.org/communicationorganisation/5583

DOI : 10.4000/communicationorganisation.5583

ISBN : 979-10-300-0146-4

ISSN : 1775-3546

Éditeur

Presses universitaires de Bordeaux

Édition imprimée

Date de publication : 1 juin 2017

Pagination : 157-174

ISBN : 979-10-300-0162-4

ISSN : 1168-5549

Référence électronique

Frédéric Compin, «Analyse du discours des acteurs en charge de la lutte contre la criminalité financière », Communication et organisation [En ligne], 51 | 2017, mis en ligne le 01 juin 2020, consulté le 02 janvier 2021. URL : http://journals.openedition.org/communicationorganisation/5583 ; DOI https://doi.org/10.4000/communicationorganisation.5583 


\title{
Analyse du discours des acteurs en charge de la lutte contre la criminalité financière
}

\author{
Frédéric Compin'
}

Nous partons du constat selon lequel la fraude fiscale induit un préjudice budgétaire pour les États, caractérisable en termes de déficit du financement des politiques sociales et aides individualisées accordées aux plus défavorisés. Évaluée entre 50 et 80 milliards d'euros en France, la fraude fiscale remet non seulement en cause les équilibres sociaux, mais implique de trouver les ressources budgétaires manquantes auprès des classes moyennes captives de l'impôt.

Dans le cadre d'une étude visant à percevoir et analyser les réponses d'acteurs ${ }^{2}$ en charge de la lutte contre les organisations facilitant et développant la criminalité financière, des entretiens semi-directifs ont été menés auprès de quarante-quatre magistrats, haut-fonctionnaires, agents des finances publiques et commissaires aux comptes ${ }^{3}$. Lexploitation de ces entretiens a fait l'objet d'une analyse de contenu réalisée grâce au logiciel Sphinx ${ }^{4}$.

L'analyse langagière des réponses apportées par les acteurs luttant contre la criminalité financière permet de dégager à la fois les orientations générales en matière de lutte contre le blanchiment de capitaux et la fraude fiscale, une perception générale sur l'état des moyens dont ils disposent, un sentiment d'insatisfaction inhérent à la réponse des pouvoirs publics et d'élaborer un constat objectif sur les normes en vigueur.

Étudier une langue comme le rappelle J. R. Searle, c'est émettre «l'hypothèse selon laquelle parler une langue c'est adopter une forme de comportement régie par des règles » (Searle 1996 : 52). Chaque personne interviewée s'est

1 Professeur en classe préparatoire au diplôme de comptabilité et gestion (DCG) au Lycée Robert Doisneau de Corbeil-Essonnes. Docteur en droit-EHESS, en sciences de gestion - CNAM et en sociologie à l'Université d'Evry val d'Essonne. Habilité à diriger des recherches ; compin.frederic@ ${ }_{\text {wanadoo.fr }}$

2 Voir Annexe n 1 : Support de l'analyse : Méthodologie générale.

3 La démarche épistémologique développée en l'espèce porte sur des enquêtes de terrain conduites sous forme d'entretiens semi-directifs, traitées à l'aide du logiciel « Sphinx iQ2 » et interprétées sur la base de la théorie des actes de langage.

4 Voir Annexe $n^{\circ} 2$ : Mode opératoire du traitement des entretiens par le logiciel Sphinx.

L'auteur remercie Madame Myriam Martins pour la mise en forme de cet article. 
exprimée au regard des us et coutumes de sa fonction, profession et règles professionnelles en vigueur. Les réponses obtenues ont été élaborées autour de l'hypothèse centrale : La criminalité financière est-elle un crime ordinaire ? Cette hypothèse se déclinant autour de trois axes majeurs : La fraude fiscale est-elle la résultante des déviances économiques ? Les criminels financiers exploitent-ils les failles normatives des systèmes de régulation ? Le système bancaire et financier facilite-t-il le blanchiment de capitaux ?

L'exploitation de ces hypothèses conduit à mettre sous tension l'existence d'un cadre normatif permissieux permettant le développement d'actes de criminalité financière (1) en identifiant le contexte spécifique et attitudes comportementales des criminels financiers (2).

\section{Mise sous tension d'un cadre normatif permissieux ${ }^{5}$ permettant le développement d'actes de criminalité financière}

Analyser le discours d'acteurs en charge de la lutte contre la criminalité financière requiert d'accepter une part de subjectivité et d'émotion tant la thématique abordée dépasse les simples aspirations individuelles pour s'inscrire dans une démarche sociétale.

J. Searle relève en effet que :

Tout ce qui dépend de l'observateur contiendra nécessairement un élément de subjectivité ontologique, parce que son existence dépend de l'attitude ou de l'intentionnalité des observateurs, participants, agents, etc., du phénomène en question. C'est un point crucial pour les sciences sociales : la relativité par rapport à l'observateur, en d'autres termes la subjectivité ontologique des phénomènes, nempêche pas leur étude en termes d'ontologie épistémique. Ainsi, par exemple, l'existence de l'argent est relative à l'observateur, conférant à l'argent une dimension de subjectivité ontologique, ce qui n'interdit pas pour autant à l'économie dêtre une science objective ; elle le peut. Pour répondre à votre question en particulier - peut-il y avoir une institution dépendante de l'observateur tout en étant ontologiquement objective ? - la réponse est non, parce que la dépendance ontologique à l'observateur implique un élément de subjectivité dans l’ontologie, et par conséquent, elle est ontologiquement subjective (Monnet, Navarro 2009).

Partant de l'analyse développée par J. L. Austin où le langage ne sert pas seulement à décrire la réalité. "En opposition à cette conception traditionnelle "vériconditionnaliste", qui appelle l'illusion descriptive, selon laquelle les énoncés auraient la proprité dềtre vrais ou faux (...) J. L. Austin défend l'idée d'une fonction du langage susceptible d'agir sur la réalité et d'accomplir certains actes. »(Gramaccia 2001: 228) Les acteurs interviewés s'inscrivent dans cette logique dans la mesure où leurs énoncés se veulent à

5 Néologisme développé par l'auteur pour mettre en évidence un cadre normatif institutionnel permis et improductif pour lutter contre la criminalité financière. 
la fois constatifs et performatifs afin de produire un effet sur les auditeurs. En d'autres termes, ces acteurs recherchent à la fois des conditions de vérité dans la délivrance de leur message mais également des conditions de satisfaction.

Le verbatim inhérent aux entretiens intégrés dans la présente analyse recoupe à la fois trois catégories d'actes de langage, des actes locutionnaires, correspondant au fait de dire quelque chose, des actes illocutionnaires accomplis en disant quelque chose et des actes perlocutionnaires accomplis par le fait de dire quelque chose 6 . À titre d'exemple, l'une des personnes interviewées déclare, au sujet de la fraude fiscale, dans le cadre d'un acte locutionnaire : "Ces chiffres ne sont bien évidemment que des ordres de grandeur ». Une autre, au sujet de la formation des agents des finances publiques, énonce dans le cadre d'un acte de langage perlocutionnaire que : "Par contre, je suis convaincu que les agents des finances publiques sont confrontés à des difficultés matérielles liées à la priorité accordée à une gestion de masse, aux relances à effectuer. » Une troisième déclare dans le cadre d'un acte de langage illocutionnaire que : "Il n'y a rien de plus facile que de réaliser une fausse opération sur un marché financier ayant l'apparence d'une vraie opération financière comme il s'en fait des milliards par jours à la surface de la planète et qui permettent de dissimuler des flux. »

Lintégration de ces énoncés repris exhaustivement permet en l'espèce l'élaboration de nuages de mots interprétables comme des unités minimales de base de la communication linguistique.

Le nuage de mots et groupes nominaux obtenu couvre plus des trois quarts du corpus.

Le discours tenu par les acteurs luttant contre la criminalité financière se décline autour de quatre thèmes ${ }^{7}$, l'impôt, la finance, la norme et le crime couvrant trois axes, la perception de la fraude fiscale par les acteurs en charge de lutter contre ce fléau, le contournement de la norme par des criminels financiers par nature déviants et le développement de la criminalité financière dans un système dérégulé.

Les acteurs interrogés expriment une opinion teintée de déception, un sentiment d'offense à leurs fonctions et un sentiment d'intérêt de l'opinion publique à la mission qu'ils exercent.

6 D'après ibid., p. 230.

7 Voir Annexe n 3 : Approche thématique. 

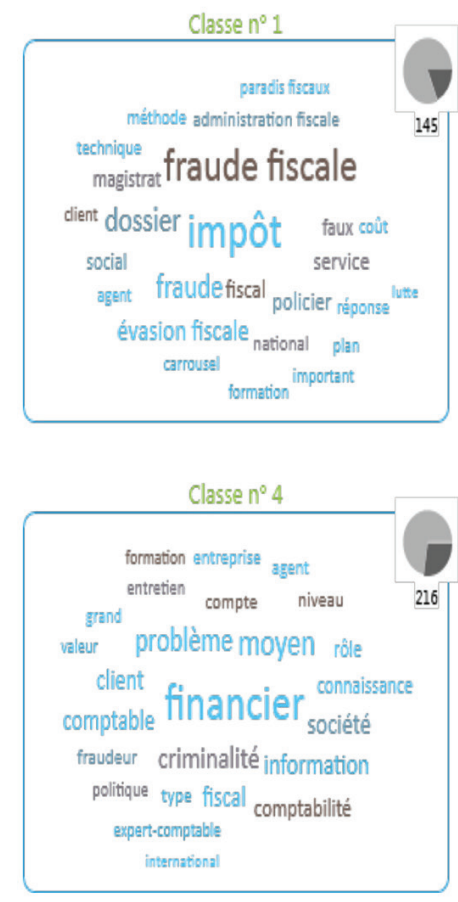

Figure 1: Répartition thématique
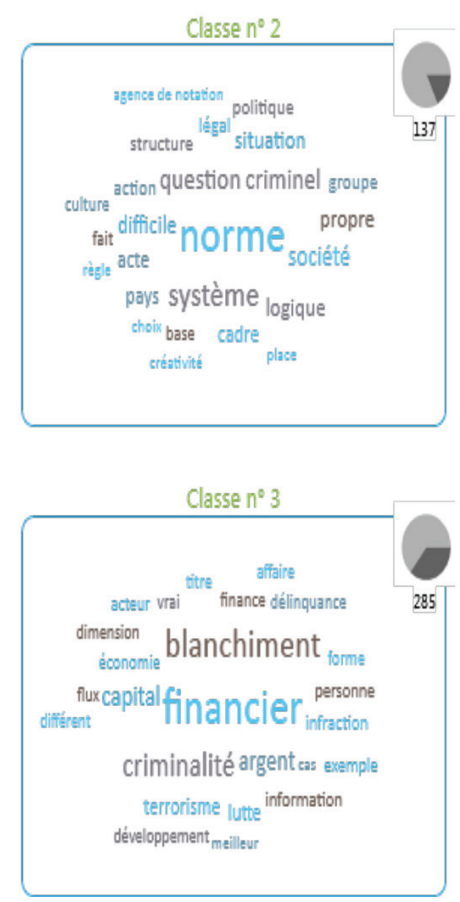

Corpus couvert à $78,3 \%$

Source : Paramétrage du logiciel réalisé par l'auteur, données recueillies par l'auteur, configuration graphique du nuage de mots réalisée par le logiciel «Sphinx»

\section{Premier axe: Perception de la fraude fiscale}

On constate que de façon générale, le terme « impôt » est mis en relation avec la «fraude fiscale », «l'évasion fiscale » et les «paradis fiscaux ». Ce constat est objectif, essentiellement réalisé par des agents des finances publiques. Il présente un intérêt vif pour les personnes interrogées en termes de manque à gagner pour les finances publiques.

Le corpus lexical repris sous forme de « verbatim » est d'ordre constatif. Les actes de langage sont d'essence locutoire.

Le tableau suivant illustre les opinions positives et négatives émises par les acteurs interviewés au sujet de la fraude fiscale ${ }^{8}$.

8 Ce tableau est produit par le logiciel Sphinx en fonction des paramètres choisis, décrits notamment dans le cadre de l'annexe $n^{\circ} 2$ : Mode opératoire du traitement des entretiens par le logiciel Sphinx. Un "verbatim » composé de groupes nominaux et de phrases est associé à des variables « sentiments » classées en deux catégories, positives ou négatives. 
Opinions positives

- Les chiffres de la fraude fiscale ont été largement médiatisés (fraude fiscale)

- Les vrais fraudeurs utilisent leurs droits de circuler librement (Comportement)

- Par contre, ils sont confrontés à des difficultés matérielles liées à la priorité accordée à une gestion de masse, aux relances à effectuer (formation)

Opinions négatives

- On se retrouve dans des situations très limites pour parvenir à atteindre leurs objectifs (fraude fiscale)

- Certaines formes de défiscalisation sont plus dangereuses que l'évasion fiscale elle-même car légale (fraude fiscale)

- Une certaine fois la frontière entre bonne foi, mauvaise foi et fraude organisée est ténue (fraude fiscale)

- Du plus simple contrôle sur pièces effectué au plus complexe contrôle externe réalisé (fraude fiscale)

La dichotomie entre réponses positives et négatives relève de la perception de contrôle de la situation ou au contraire d'impuissance face à l'organisation des fraudeurs.

Le syntagme nominal de fraude fiscale est à rapprocher de celui d'évasion fiscale, perçu également négativement au travers du verbatim.

- Je n'ai aucun doute sur le fait qu'il n'y a pas une distinction à faire entre le blanchiment d'un trafic quelconque et le blanchiment de fraude fiscale (Évasion fiscale)

- Il y a eu dans le temps une distinction qui repose sur une logique développée par certaines juridictions non coopératives, au Lichtenstein ou en en Suisse, il ny a pas d'incrimination pénale pour la fraude fiscale ou l'évasion fiscale ; (Évasion fiscale)

- On n'est pas dans la situation de la Grèce, mais on voit bien que la culture de l'évasion fiscale ou de l'évasion de l'impôt doit disparaître pour que le pays se relève et survive (Évasion fiscale)

- L'acte devrait être prohibé sachant cependant que la question de la prohibition est par nature un choix politique basé sur des moyens pour l'interdire (Evasion fiscale)

- Au sein de l'administration française, la fraude ou l'évasion fiscale, les deux concepts sont liés (Évasion fiscale)

Deuxième axe : Les déviances normatives opérées par les criminels financiers

Le rapport à la norme des acteurs interrogés n'entraîne pas de sentiment spécifique. L'une des phrases clés du verbatim relève la dimension locutoire de l'exposé. Il s'agit d'informer et de faire prendre conscience aux auditeurs 
comment procèdent les auteurs d'infractions financières, "Les auteurs qui répondent à des normes propres à un groupe culturel ou social nient l'existence d'une collectivité politique supérieure, légitime à leur imposer des normes qui peuvent être contraires aux leurs ou à leurs intérêts (Criminalité financière) »; "Il a conscience qu'il dépasse non seulement la norme sociale mais qu'il ne respecte pas la loi en commettant une infraction (Norme) »;

Comme le relève J. Searle :

C'est la capacité langagière et, partant, la capacité à créer des faits institutionnels, qui nous permet de créer des structures telles que les gouvernements, les relations économiques, le mariage, la propriété privée, dont nous pouvons attendre une certaine stabilité opératoire, parce que les participants d'une même institution reconnaissent qu'ils ont des raisons d'agir indépendantes de leurs inclinations immédiates?

Il s'avère cependant que l'association des mots «État » et « norme » sont révélateurs d'une perception d'impuissance face aux déviances normatives. Le discours demeure en l'espèce constatif. L'acte de langage des acteurs interviewés est également illocutoire en étant verdictif, c'est-à-dire élaboré à partir d'un corpus de termes juridiques :

- L'évolution de la corruption traduit le non-respect des lois et règlements facilité par des mesures de déréglementation des marchés (État),

- Les États sont dans des rapports de forme hiérarchisée démontrant l'existence de très fortes différences entre eux (État),

- Cette impuissance est d'ailleurs encouragée par la réglementation des marchés financiers dès 1967 (État).

Troisième axe: Le développement de la criminalité financière dans un système financier dérégulé

Par contre lorsque les acteurs interrogés sont confrontés au thème concret du blanchiment de capitaux dans un système financier de type capitaliste, les réponses revêtent une dimension perlocutoire en faisant ressortir un sentiment d'offense à leurs fonctions exercées et une déception inhérente aux moyens dont ils disposent pour mener leurs missions. Le verbatim est éloquent en la matière :

- C'est vrai que lorsquon parle blanchiment et criminalité financière, on ne veut pas se cantonner dans légalité criminalité financière égale délinquance économique ou délinquance des financiers (Régulation),

9 É. Monnet, P. Navarro, op. cit. 
- Il n'y a rien de plus facile que de réaliser une fausse opération sur un marché financier ayant l'apparence d'une vraie opération financière comme il s'en fait des milliards par jours à la surface de la planète et qui permettent de dissimuler des flux,

- On peut se trouver dans trois situations différentes, la situation classique qui est celle d'une délinquance mise en œuvre à partir de la personnalité de l'acteur qui s'implique dans la transgression de la norme,

- Il s'ensuit que le capitalisme, en étant à l'origine de nouvelles formes de prédation, constitue un facteur d'évolution de la criminalité (Régulation),

- Il existe deux types de situation, la fraude individuelle dont l'exemple type est Madoff et la fraude institutionnelle (Comportement),

- C'est l'autonomie de la finance, des organismes financiers qui s'expriment dans les formes institutionnelles des rapports sociaux spécifiques (Régulation).

Le discours tenu par les acteurs en charge de la lutte contre la criminalité financière est d'essence institutionnelle. Ils posent en l'espèce la problématique de la régulation du système financier comme la pierre angulaire de leur action. Comme le mentionne G. Gramaccia, s'appuyant sur l'ouvrage publié par R. Lourau en 1970, L'analyse institutionnelle, une distinction doit être opérée entre : "Sa "face institutionnelle" proprement dite, qui doit être saisie dans sa composante symbolique, et sa "face matérielle", sa "face organisationnelle", qui correspond à la mise en œuvre des contraintes pratiques de la coordination et de la coopération ${ }^{10}$ ».

\section{Contexte spécifique et attitudes comportementales des criminels financiers}

Le traitement de la variable de contexte ${ }^{11}$ permet de comprendre les différences entre les répondants. Elle permet de segmenter le corpus selon plusieurs catégories, comme l'âge, le sexe, ou son département / service ou autre $^{12}$.

Il est permis d'observer premièrement que la variable « comportement » des criminels financiers est à corréler avec la variable « fraude fiscale » et « évasion fiscale ». Les nuages de mots se rejoignent autour d'une sémantique commune. Les acteurs interviewés relèvent que le comportement du délinquant fraudeur doit s'expliquer dans un contexte économique donné. Le verbatim spécifique à ce contexte en témoigne ; «J'aborde ces questions de fraude, de criminalité financière en faisant abstraction de la personne et des mobiles des acteurs "; "Il existe deux types de situations, la fraude individuelle dont l'exemple type est Madoff et la fraude institutionnelle »; " En revanche ce qui m’intéresse,

10 G. Gramaccia, op. cit, p. 52-53.

11 Voir Annexe n 4 : Approche contextuelle.

12 Voir Annexe n 5 : Mode opératoire de l'approche contextuelle. 


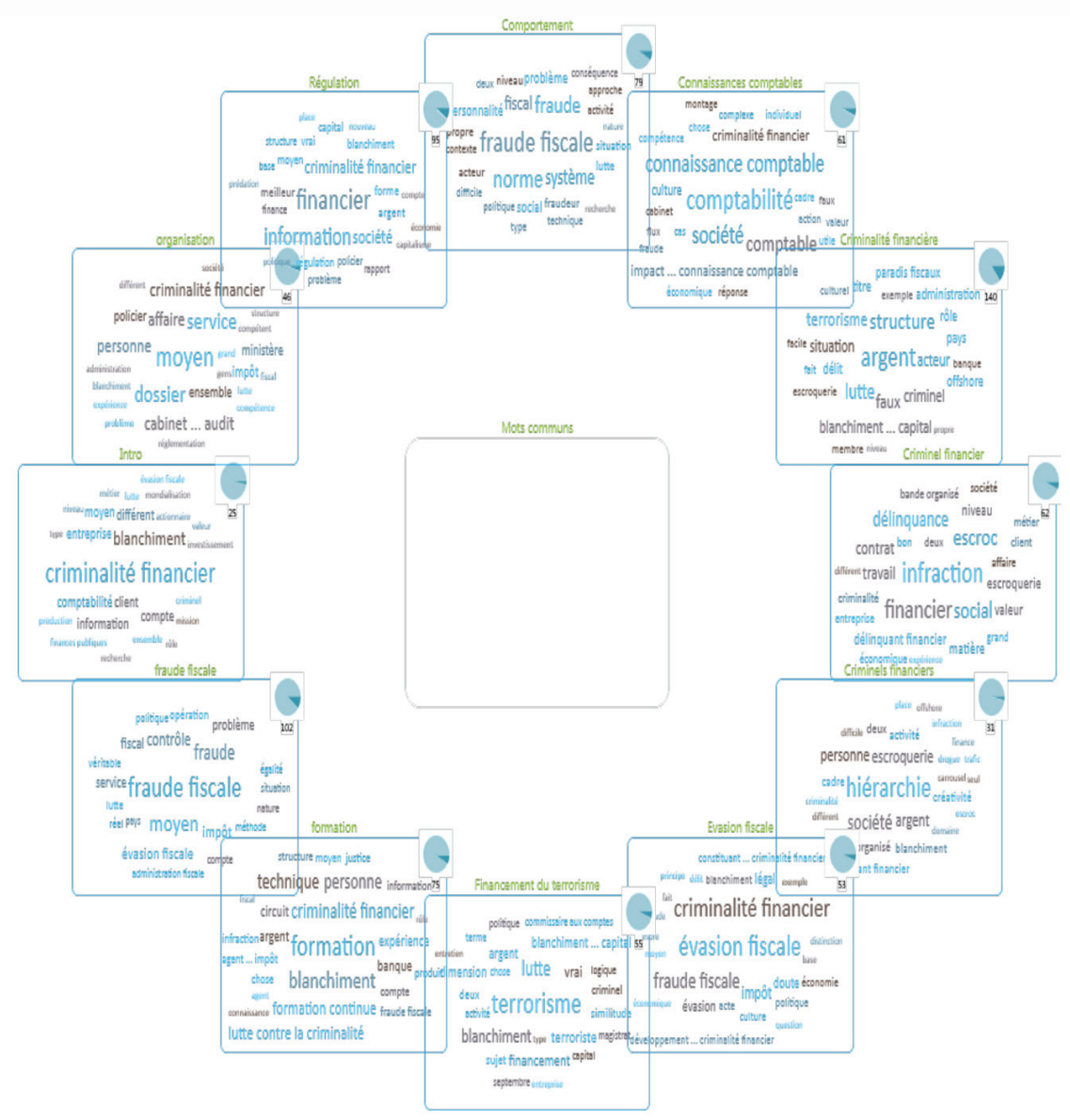

Figure 2 - Contexte spécifique.

Source : Paramétrage du logiciel réalisé par l'auteur, données recueillies par l'auteur, configuration graphique du nuage de mots réalisée par le logiciel « Sphinx ».

dans les domaines où il existe une logique criminelle, ce qu'il faut rechercher c'est en quoi la transgression de règles est utile au système lui-même "; « La seule approche possible c'est d'étudier le système pour savoir comment il instrumentalise la fraude pour assurer son émergence et son développement. »

La seconde observation inhérente au contexte porte sur la variable " organisation ». Les acteurs en charge de la lutte contre la criminalité financière déclinent leurs missions autour de deux axes, la lutte contre le blanchiment de capitaux et la fraude fiscale. Ils prennent soin de bien les 
dissocier du « financement du terrorisme»" ${ }^{13}$. Les nuages de mots relatifs au contexte « organisation » sont à rapprocher des contextes « connaissances » et «formation ». Ils rejoignent le contexte plus global « d'administration fiscale ». Les phrases clés retenues lors de ces entretiens semi-directifs constituent essentiellement des actes de langage perlocutoires ; leurs auteurs veulent alerter l'auditeur des difficultés rencontrées pour accomplir souverainement leurs missions. Le verbatim spécifique à ce contexte l'illustre catégoriquement ${ }^{14}$, "Souvent on voit que l'on a des dossiers de carrousel de TVA et les avocats de l'administration fiscale ne sont pas constitués»; "Le dossier est fini sans que l'on obtienne de réponses »; "Cela m’échappe en qualité de magistrat instructeur mais c'est un travail qui doit être mené avec le parquet »; «Le collègue qui traite le dossier ne sera pas informé des suites qui seront données »; "Ce serait utile que ces personnes-là viennent faire de la formation continue pour les agents compétents dans les services concernés »; «L'action de l'État ne traite pas forcément l'ensemble du problème »; «En déclinant cette question, on rencontre des personnes qui disent qu'elles souffrent d'un manque de moyens et d'absence de volonté politique "; "Nous sommes confrontés à deux types de problèmes, des moyens humains insuffisants notamment et une absence de réelle volonté politique ».

La troisième observation porte sur le niveau des responsabilités. Les acteurs en charge de la lutte contre la criminalité financière placent la régulation de l'information financière comme une donnée contextuelle centrale leur permettant d'assumer leur mission avec efficacité. Les mots associés au contexte régulation sont « information, information des sociétés, information financière ». Le discours dominant repose sur une mise en perspective de l'état des normes en vigueur « Les normes émises rentrent en conflit avec les normes globales et les acteurs mettent en œuvre des modes de fonctionnement destinés à préserver l'intégrité du système dans lequel ils évoluent et à transgresser les normes dans la société légale ». Le mot «banques » est ici structurant et transgressif dans l'esprit des personnes interviewées car connoté à responsabilité de cet agent institutionnel. Le discours dominant porte sur la relation entre le secteur bancaire et l'émergence de la criminalité financière "Il convient plutôt de rechercher des niveaux de responsabilité du côté des banques, des fonds de pension, des fonds mutuels actionnaires des hedge funds dont l'action déstabilise les marchés »; " Grâce aux innombrables innovations financières portant sur des produits complexes, les banques se sont dotées de moyens considérables pour favoriser une opacité totale génératrice indirectement d'optimisation fiscale »; « La logique de valorisation du capital

13 Frédéric Compin, "Financement du terrorisme et blanchiment de capitaux : Liaisons dangereuses ou manipulations d'État ?", L'Homme et la société, n 199, 2016/1, janvier-mars 2016, p. 159-172.

14. Les énoncés repris entre guillemets sont extraits d'entretiens réalisés par l'auteur auprès des acteurs en charge de la lutte contre la criminalité financière. 
génère ses propres dérives ». " La finance sécrète la criminalité financière en favorisant la prédation dans de nombreux domaines de biens publics comme la connaissance par exemple ».

\section{Conclusion}

La synthèse de l'examen du discours des acteurs, interviewés, en charge de la lutte contre la criminalité financière est élaborée à partir d'un mode de représentation graphique de tableaux de contingence. Reprenant l'approche de G. Lapassade : "L'analyseur est tout ce qui fait surgir la vérité de ce qui est caché ; tout, c'est-à-dire groupe, individu, situation, évènement, scandale » (Gramaccia: 55) L'analyse factorielle des correspondances permet, en effet à partir de tris croisés multiples, « de rassembler en un ou plusieurs graphes, la plus grande partie possible de l'information contenue dans le tableau, en s'attachant non pas aux valeurs absolues, mais aux correspondances entre les caractères, c'est-à-dire aux valeurs relatives » (Lagarde 1983: 54).

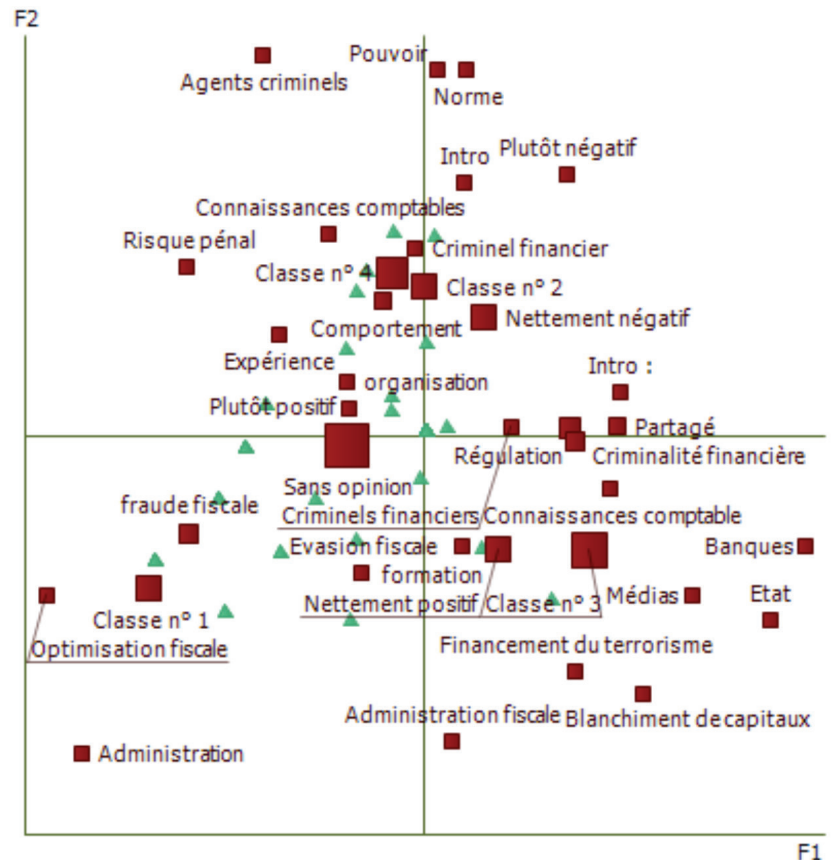

Figure 3 : Représentation graphique de l'AFC.

Source : Paramétrage du logiciel réalisé par l'auteur, données recueillies par l'auteur, configuration graphique du nuage de mots réalisée par le logiciel « Sphinx ». 
On perçoit que les termes " organisation ", " régulation » et " criminalité financière » sont très proches de la moyenne. Ils signifient que la régulation des organisations permet de lutter contre la criminalité financière. Ces « organisations » nationales, internationales, professionnelles sont perfectibles donc régulables. Proche de la moyenne, c'est-à-dire à l'intersection des axes les termes « comportement », " organisation », « expérience » et « connaissances comptables » se rejoignent pour signifier que les criminels financiers œuvrent dans des organisations leur permettant d'exploiter leurs connaissances comptables en faisant fructifier leur expérience délictuelle.

Plus excentré, le terme de criminel financier n'est pas caractéristique de l'ampleur de la lutte à mener car trop anecdotique pour refléter une vérité avérée. Cette analyse factorielle des correspondances permet de mesurer à quel point la lutte contre la criminalité financière relève d'une dimension macro-sociale nécessitant de rendre perfectible les organisations en charge de lutter contre le blanchiment de capitaux et la fraude fiscale. Il importe donc de dépasser une approche exclusivement orientée vers le criminel financier comme donnée révélatrice d'un système criminogène.

\section{BIBLIOGRAPHIE}

GRAMACCIA G., Les actes de langage dans les organisations, L'Harmattan, mars 2001, Paris, p. 228-230.

LAGARDE J. de., Initiation à l'analyse des données, Dunod, 1983, Paris, p. 54.

LAPASSADE G., L'arpenteur, EPI, 1971, p. 23 cité par Gramaccia G., op. cit., Paris, p. 55 .

http://infos.lesphinx.eu/docquali/fr/presentation-de-sphinx-quali/modesoperatoires/import-de-donnees-terrain.html\#directif (Consulté le 6 mai 2016)

http://infos.lesphinx.eu/docquali/fr/presentation-de-sphinx-quali/glossaire. html\#varContexte (Consulté le 6 mai 2016)

http://infos.lesphinx.eu/docquali/fr/protocole-de-codification/modes-operatoires/ codebook-automatique.html (Consulté le 6 mai 2016)

http://infos.lesphinx.eu/docquali/fr/protocole-synthese.html (Consulté le 6 mai 2016)

MONNET É., NAVARRO P., « Les institutions sont-elles dans la tête ? Entretien avec John Searle », Tracés. Revue de Sciences humaines [En ligne], 17 | 2009, mis en ligne le 30 novembre 2011. URL : https://traces.revues.org/4270 (Consulté le 19 mai 2016).

SEARLE J. R., Les actes de langage, Collection Savoir : Lettres, Herman, $1^{\text {er }}$ édition, 1972, Paris, 1996, p. 52.

Résumé : L'analyse du contenu du discours de quarante-quatre agents en charge de la lutte contre la criminalité financière repose sur trois thèmes précis, la perception de la fraude fiscale, les déviances normatives opérées par les criminels financiers et le 


\title{
$\mathrm{C} \& O \mathrm{n}^{\circ} 51$
}

développement de la criminalité financière dans un système financier dérégulé. Cette thématique est inhérente au contexte spécifique dans lequel se déroulent ces enquêtes de terrain élaborées autour d'entretiens semi-directifs. L'utilisation du logiciel d'analyse des données «Sphinx iQ2 " permet la construction de nuages de mots faisant ressortir des tendances langagières observables sous forme de thèmes dominants ou de contextes d'études.

Mots-clés : analyse de contenu, discours, criminalité financière, fraude fiscale, norme.

\begin{abstract}
This discourse and content analysis of forty four officials leading the fight against financial crime is based on three precise issues: the perception of tax evasion, the normative deviations made by financial criminals and the development of financial crime in a deregulated financial system. This theme is inherent in a specific context of field surveys based on semistructured interviews. The use of «Sphinx iQ2» data analysis software allowed the construction of word clouds highlighting language trends observable in the form of dominant themes or in the context of research studies.
\end{abstract}

Keywords: discourse and content analysis, financial crime, tax evasion, norm.

\section{Annexe $n^{\circ} 1$ : Méthodologie générale, supports de l'analyse}

La méthode développée repose sur des enquêtes de terrain.

L'enquête réalisée sous forme d'entretiens semi-directifs a été effectuée entre janvier 2011 et juillet 2015 auprès de vingt-cinq agents des finances publiques, de catégories $\mathrm{A}, \mathrm{B}$ et $\mathrm{C}$, de magistrats de lordre judiciaire, de quinze commissaires aux comptes, d'un représentant d'une grande centrale syndicale du ministère de l'Économie, des Finances et de l'Industrie et d'un haut fonctionnaire du Ministère de l'Économie, des Finances et de l'Industrie. Le panel des personnes interrogées a permis de recueillir des informations pratiques, techniques et professionnelles d'horizons connexes mais différents quant à l'exercice de la mission exercée en matière de perception des actes de criminalité financière.

\section{Annexe $n^{\circ} 2$ : Mode opératoire du traitement des entretiens par le logiciel Sphinx}

1. Intégration des entretiens directifs ou semi directifs dans un fichier unique.

2. Retranscription se fait en alternant les couples question / réponse. On utilise les balises $\mathrm{Q}$ : ou $\mathrm{R}$ : en début de ligne de chaque question ou réponse.

3. Plusieurs entretiens peuvent être retranscrits à la suite. On utilise la balise $\mathrm{E}$ : pour repérer les différents entretiens. En tête de la retranscription 
d'un entretien figurent les données caractérisant l'interview. Elles sont définies par des balises, par exemple : Nom, Prénom, Age, CSP, Date... Ces indications figurent en début de ligne. Le signe «:» sépare la balise de son contenu.

4. Ajout d'annotations pour marquer des expressions non verbales ou tout autre commentaire ne faisant pas partie du texte retranscris ;

5. Intégration de Jalons ou repères. « Lindication des différents thèmes abordés ou différentes parties de l'entretien se fait par l'ajout de repères (jalon) «>» suivi d'un mot. $»^{15}$

6. À titre d'exemple, E : Entretien avec des agents des finances publiques.

Nom : Agents des finances publiques.

CSP : Inspecteur et inspectrice des impôts

Date : 2011-2013

Mots-clés : fraude fiscale, évasion fiscale, optimisation fiscale, fraudeurs, criminels financiers, fraude, délits, crimes, lutte, efficacité, égalité, égalité de traitement, politique fiscale légalité, information, formation connaissances

$>$ Intro

L'entretien réalisé avec des agents des finances publiques, inspecteur et inspectrice des impôts, porte, d'une part sur la formation dispensée par l'École Nationale des Impôts (ENI) pour lutter contre la fraude fiscale et d'autre part sur les moyens dont les agents des impôts peuvent disposer contre les fraudeurs et contribuables adeptes de l'évasion fiscale.

$>$ formation

Q: Pensez- vous que la formation dispensée à l'ENI prépare les agents des impôts à lutter contre la fraude fiscale ? [Formation]

$\mathrm{R}$ : «Oui, je le pense parce que les spécificités du terrain sont enseignées par des formateurs disposant d'une réelle expérience, d'un savoir-faire pratique et d'une connaissance de la réalité des choses. La formation repose sur des cas concrets. Les enseignants-formateurs ont trois ans minimum d'expérience de terrain ». [Expérience]

$>$ fraude fiscale

7. Création d'un code book. "Le codebook est la grille d'analyse du corpus, en général elle contient des thèmes (idées) présents dans le corpus mais peut parfois contenir des éléments descriptifs permettant de classer les textes (source des extraits, auteur...). Cette étape permet d'obtenir des résultats quantitatifs de votre analyse (nombre de thèmes cités, répartition des thèmes etc...). Chaque élément de la grille est appelé « variable ». Il est possible de créer automatiquement un codebook grâce au moteur sémantique de Sphinx Quali. Cette fonction crée la grille puis code automatiquement les observations. Le codebook automatique contient

15 http://infos.lesphinx.eu/docquali/fr/presentation-de-sphinx-quali/modes-operatoires/import-de-donneesterrain.html\#directif (Consulté le 6 mai 2016) 
les concepts présents dans le corpus et chaque observation est codée pour chaque concept y faisant référence. $»^{16}$

8. Mise en place d'un protocole de synthèse. «Le protocole de Synthèse permet de visualiser les résultats des analyses textuelles et sémantiques, et de préparer leur restitution. Quatre types de synthèses peuvent être affichés : Synthèse globale, orientation et sentiments, analyse par contexte, analyse de contenu. Toutes les modifications effectuées dans le corpus et le codage sont prises en compte lors de la création des synthèses. ${ }^{17}$

\section{Annexe n³: Approche thématique}

\begin{tabular}{|c|c|c|c|c|c|c|c|c|}
\hline & Effectifs & $\begin{array}{c}\text { Longueur } \\
\text { moyenne }\end{array}$ & Les 5 mots spécifiques & $\begin{array}{l}\text { Concept } \\
\text { spécifique }\end{array}$ & $\begin{array}{l}\text { Sentiment } \\
\text { spécifique }\end{array}$ & $\begin{array}{l}\text { Contexte } \\
\text { spécifique }\end{array}$ & $\begin{array}{l}\text { Orientation } \\
\text { spécifique }\end{array}$ & $\begin{array}{l}\text { Indice de } \\
\text { richesse } \\
\text { relative }\end{array}$ \\
\hline $\begin{array}{c}\text { Classe } \\
n^{\circ} 1\end{array}$ & 145 & 6 & $\begin{array}{l}\text { impôt - fraude fiscale } \\
\text { - dossier - fraude - } \\
\text { évasion fiscale }\end{array}$ & $\begin{array}{l}\text { Politique } \\
\text { fiscale }\end{array}$ & Intérêt & $\begin{array}{l}\text { Adminis- } \\
\text { tration }\end{array}$ & $\begin{array}{l}\text { Sans } \\
\text { opinion }\end{array}$ & 0,92 \\
\hline $\begin{array}{c}\text { Classe } \\
n^{\circ} 2\end{array}$ & 137 & 7 & $\begin{array}{l}\text { norme - système - } \\
\text { question - criminel } \\
\text { - société }\end{array}$ & $\begin{array}{l}\text { Rapport à } \\
\text { la norme }\end{array}$ & & $\begin{array}{l}\text { Agents } \\
\text { criminels }\end{array}$ & & 1,00 \\
\hline $\begin{array}{c}\text { Classe } \\
n^{\circ} 3\end{array}$ & 285 & 8 & $\begin{array}{l}\text { financier - blanchiment } \\
\text { - criminalité - capital } \\
\text { - argent }\end{array}$ & $\begin{array}{l}\text { Crimes et } \\
\text { délits }\end{array}$ & Offense & État & Partagé & 1,12 \\
\hline $\begin{array}{c}\text { Classe } \\
n^{\circ} 4\end{array}$ & 216 & 7 & $\begin{array}{l}\text { financier - moyen - } \\
\text { problème - criminalité } \\
\text { - information - ... }\end{array}$ & $\begin{array}{l}\text { Épargne } \\
\text { et gestion }\end{array}$ & Déception & $\begin{array}{l}\text { Risque } \\
\text { pénal }\end{array}$ & & 0,97 \\
\hline
\end{tabular}

La richesse d'un corpus est le nombre de mots différents d'un corpus. Si l'indice de richesse est supérieur à 1 alors les réponses de cette catégorie sont plus riches que la moyenne. À l'inverse (indice inférieur à 1), les réponses de cette catégorie sont moins riches que la moyenne.

$16 \mathrm{http} / /$ infos.lesphinx.eu/docquali/fr/protocole-de-codification/modes-operatoires/codebook-automatique. html (Consulté le 6 mai 2016)

17 http://infos.lesphinx.eu/docquali/fr/protocole-synthese.html (Consulté le 6 mai 2016) 


\section{Annexe $n^{\circ} 4$ : Approche contextuelle}

Les classes contribuent à mettre en évidence les thématiques de l'étude. Les nuages représentent les principaux mots spécifiques de chaque classe (tailles proportionnelles aux effectifs).

\begin{tabular}{|c|c|c|c|c|c|c|c|c|}
\hline & Effectifs & $\begin{array}{c}\text { Longueur } \\
\text { moyenne }\end{array}$ & $\begin{array}{l}\text { Les } 5 \text { mots } \\
\text { spécifiques }\end{array}$ & $\begin{array}{l}\text { Concept } \\
\text { spécifique }\end{array}$ & $\begin{array}{l}\text { Sentiment } \\
\text { spécifique }\end{array}$ & $\begin{array}{c}\text { Classe } \\
\text { spécifique }\end{array}$ & $\begin{array}{c}\text { Orientation } \\
\text { spécifique }\end{array}$ & $\begin{array}{l}\text { Indice de } \\
\text { richesse } \\
\text { relative }\end{array}$ \\
\hline $\begin{array}{l}\text { Adminis- } \\
\text { tration } \\
\text { fiscale }\end{array}$ & 15 & 13 & $\begin{array}{l}\text { financier - } \\
\text { blanchiment } \\
\text { - délinquance } \\
\text { - dimension } \\
\text { - criminalité } \\
\text { financier - ... }\end{array}$ & $\begin{array}{l}\text { Crimes et } \\
\text { délits }\end{array}$ & & & $\begin{array}{l}\text { Plutôt } \\
\text { positive }\end{array}$ & 1,63 \\
\hline $\begin{array}{l}\text { Agents } \\
\text { criminels }\end{array}$ & 7 & 6 & $\begin{array}{l}\text { agence de } \\
\text { notation - critère } \\
\text { - mission - } \\
\text { financier - social }\end{array}$ & MultiMedia & & $\begin{array}{l}\text { Classe } \\
n^{\circ} 2\end{array}$ & \begin{tabular}{|l} 
Sans \\
opinion
\end{tabular} & 0,74 \\
\hline Banques & 8 & 11 & $\begin{array}{l}\text { dérive - banque - } \\
\text { financier - hedge } \\
\text { funds - nouveau } \\
-\ldots\end{array}$ & $\begin{array}{c}\text { Trésorerie et } \\
\text { banques }\end{array}$ & Intérêt & $\begin{array}{l}\text { Classe } \\
n^{\circ} 3\end{array}$ & & 1,44 \\
\hline $\begin{array}{l}\text { Blanchiment } \\
\text { de capitaux }\end{array}$ & 14 & 7 & $\begin{array}{l}\text { terroriste - } \\
\text { flux - attentat } \\
\text { - financier - coût } \\
-\ldots\end{array}$ & $\begin{array}{c}\text { Degré } \\
\text { d'aisance - ... }\end{array}$ & & $\begin{array}{l}\text { Classe } \\
n^{\circ} 3\end{array}$ & & 0,89 \\
\hline $\begin{array}{l}\text { Comporte- } \\
\text { ment }\end{array}$ & 79 & 9 & $\begin{array}{l}\text { fraude fiscale - } \\
\text { norme - fraude } \\
\text { - système - fiscal }\end{array}$ & $\begin{array}{l}\text { Crimes et } \\
\text { délits - ... }\end{array}$ & & $\begin{array}{c}\text { Classe } \\
n^{\circ} 2\end{array}$ & & 1,08 \\
\hline $\begin{array}{l}\text { Connais- } \\
\text { sances } \\
\text { comptable }\end{array}$ & 23 & 7 & $\begin{array}{l}\text { financier - circuit } \\
\text { - financement } \\
\text { - mondialisation } \\
\text { - demande }\end{array}$ & Combat & & & $\begin{array}{l}\text { Nettement } \\
\text { positive }\end{array}$ & 0,91 \\
\hline $\begin{array}{l}\text { Connais- } \\
\text { sances } \\
\text { comptables }\end{array}$ & 61 & 6 & $\begin{array}{l}\text { comptabilité } \\
\text { - société - } \\
\text { connaissance } \\
\text { comptable } \\
\text { - comptable } \\
\text { - impact ... } \\
\text { connaissance } \\
\text { comptable - ... }\end{array}$ & $\begin{array}{l}\text { Épargne et } \\
\text { gestion }\end{array}$ & Offense & $\begin{array}{l}\text { Classe } \\
n^{\circ} 4\end{array}$ & \begin{tabular}{|l} 
Sans \\
opinion
\end{tabular} & 0,77 \\
\hline $\begin{array}{l}\text { Criminalité } \\
\text { financière }\end{array}$ & 140 & 7 & $\begin{array}{l}\text { argent - structure } \\
\text { - lutte - faux }\end{array}$ & $\begin{array}{l}\text { Crimes et } \\
\text { délits }\end{array}$ & Offense & $\begin{array}{l}\text { Classe } \\
n^{\circ} 3\end{array}$ & Partagé & 0,90 \\
\hline
\end{tabular}




\begin{tabular}{|c|c|c|c|c|c|c|c|c|}
\hline & Effectifs & $\begin{array}{c}\text { Longueur } \\
\text { moyenne }\end{array}$ & $\begin{array}{l}\text { Les } 5 \text { mots } \\
\text { spécifiques }\end{array}$ & $\begin{array}{l}\text { Concept } \\
\text { spécifique }\end{array}$ & $\begin{array}{l}\text { Sentiment } \\
\text { spécifique }\end{array}$ & $\begin{array}{c}\text { Classe } \\
\text { spécifique }\end{array}$ & $\begin{array}{c}\text { Orientation } \\
\text { spécifique }\end{array}$ & $\begin{array}{l}\text { Indice de } \\
\text { richesse } \\
\text { relative }\end{array}$ \\
\hline $\begin{array}{l}\text { Criminel } \\
\text { financier }\end{array}$ & 62 & 5 & $\begin{array}{l}\text { infraction } \\
\text { - financier - } \\
\text { escroc - social } \\
\text { - délinquance }\end{array}$ & $\begin{array}{l}\text { Crimes et } \\
\text { délits }\end{array}$ & Blâmer & & $\begin{array}{l}\text { Plutôt } \\
\text { négative }\end{array}$ & 0,67 \\
\hline $\begin{array}{l}\text { Criminels } \\
\text { financiers }\end{array}$ & 31 & 6 & \begin{tabular}{|l|} 
hiérarchie \\
- société \\
- escroquerie - \\
personne - argent
\end{tabular} & \begin{tabular}{|l} 
Degré \\
d'aisance - ...
\end{tabular} & & $\begin{array}{c}\text { Classe } \\
n^{\circ} 2\end{array}$ & & 0,75 \\
\hline État & 7 & 8 & $\begin{array}{l}\text { évolution - } \\
\text { corruption - rôle } \\
\text { - loi - mesure - ... }\end{array}$ & $\begin{array}{l}\text { Degré de } \\
\text { libéralisme }\end{array}$ & & $\begin{array}{l}\text { Classe } \\
n^{\circ} 3\end{array}$ & & 0,97 \\
\hline Évasion fiscale & 53 & 7 & $\begin{array}{l}\text { évasion fiscale } \\
\text { - criminalité } \\
\text { financier - fraude } \\
\text { fiscale - impôt - } \\
\text { légal - ... }\end{array}$ & $\begin{array}{l}\text { Politique } \\
\text { fiscale }\end{array}$ & & $\begin{array}{c}\text { Classe } \\
n^{\circ} 1\end{array}$ & & 0,93 \\
\hline Expérience & 7 & 7 & $\begin{array}{l}\text { évasion fiscale } \\
\text { - problème } \\
\text { - information - } \\
\text { criminalité } \\
\text { financier - } \\
\text { question - ... }\end{array}$ & Difficulté - ... & & & & 0,91 \\
\hline formation & 75 & 7 & $\begin{array}{l}\text { formation - } \\
\text { blanchiment } \\
\text { - criminalité } \\
\text { financier } \\
\text { - technique - } \\
\text { personne }\end{array}$ & $\begin{array}{l}\text { Trésorerie et } \\
\text { banques }\end{array}$ & & & & 0,95 \\
\hline fraude fiscale & 102 & 7 & $\begin{array}{l}\text { fraude fiscale - } \\
\text { moyen - fraude - } \\
\text { contrôle - impôt } \\
-\ldots\end{array}$ & $\begin{array}{l}\text { Politique } \\
\text { fiscale }\end{array}$ & Peur & $\begin{array}{c}\text { Classe } \\
n^{\circ} 1\end{array}$ & & 0,88 \\
\hline Médias & 3 & 10 & $\begin{array}{l}\text { personne } \\
\text { - opération - } \\
\text { cas - opinion } \\
\text { publique }\end{array}$ & $\begin{array}{l}\text { Art pictural } \\
-\ldots\end{array}$ & Ennui & & & 1,23 \\
\hline Norme & 6 & 8 & $\begin{array}{l}\text { norme - loi } \\
\text { - infraction } \\
\text { - délinquant } \\
\text { financier }\end{array}$ & $\begin{array}{l}\text { Crimes et } \\
\text { délits }\end{array}$ & Intérêt & & & 1,00 \\
\hline $\begin{array}{l}\text { Optimisation } \\
\text { fiscale }\end{array}$ & 22 & 7 & $\begin{array}{l}\text { impôt - client - } \\
\text { bureau ... achat } \\
\text { - produit - fiscal }\end{array}$ & $\begin{array}{l}\text { Politique } \\
\text { fiscale }\end{array}$ & Louer & $\begin{array}{c}\text { Classe } \\
n^{\circ} 1\end{array}$ & $\begin{array}{l}\text { Sans } \\
\text { opinion }\end{array}$ & 0,94 \\
\hline
\end{tabular}




\begin{tabular}{|c|c|c|c|c|c|c|c|c|}
\hline & Effectifs & $\begin{array}{c}\text { Longueur } \\
\text { moyenne }\end{array}$ & $\begin{array}{l}\text { Les } 5 \text { mots } \\
\text { spécifiques }\end{array}$ & $\begin{array}{c}\text { Concept } \\
\text { spécifique }\end{array}$ & $\begin{array}{l}\text { Sentiment } \\
\text { spécifique }\end{array}$ & $\begin{array}{c}\text { Classe } \\
\text { spécifique }\end{array}$ & $\begin{array}{c}\text { Orientation } \\
\text { spécifique }\end{array}$ & $\begin{array}{c}\text { Indice de } \\
\text { richesse } \\
\text { relative }\end{array}$ \\
\hline organisation & 46 & 7 & $\begin{array}{l}\text { moyen - service } \\
\text { - dossier - affaire } \\
\text { - personne - ... }\end{array}$ & $\begin{array}{l}\text { Politique } \\
\text { fiscale }\end{array}$ & Évaluation & $\begin{array}{c}\text { Classe } \\
n^{\circ} 4\end{array}$ & & 0,88 \\
\hline Pouvoir & 24 & 8 & \begin{tabular}{|l|} 
politique - \\
juridiction \\
- volonté - \\
question - norme
\end{tabular} & $\begin{array}{l}\text { Politique } \\
\text { fiscale }\end{array}$ & Louer & $\begin{array}{c}\text { Classe } \\
n^{\circ} 2\end{array}$ & $\begin{array}{l}\text { Plutôt } \\
\text { positive }\end{array}$ & 0,97 \\
\hline Régulation & 95 & 8 & $\begin{array}{l}\text { financier - } \\
\text { information } \\
\text { - société } \\
\text { - criminalité } \\
\text { financier - } \\
\text { meilleur - ... }\end{array}$ & $\begin{array}{l}\text { Communi- } \\
\text { cation - ... }\end{array}$ & Évaluation & $\begin{array}{c}\text { Classe } \\
n^{\circ} 3\end{array}$ & & 0,98 \\
\hline
\end{tabular}

La richesse d'un corpus est le nombre de mots différents d'un corpus. Si l'indice de richesse est supérieur à 1 alors les réponses de cette catégorie sont plus riches que la moyenne. À l'inverse (indice inférieur à 1), les réponses de cette catégorie sont moins riches que la moyenne.

\section{Annexe $n^{\circ} 5$ : Mode opératoire de l'approche contextuelle}

La variable de contexte doit être une variable fermée unique ou fermée échelle. ${ }^{18}$

Cette vue est calculée en fonction de la variable de contexte sélectionnée et met en évidence :

- Le vocabulaire spécifique des différentes catégories de contextes (sources, partie de document, identité des répondants...), présenté sous forme de nuages de mots dans les pavés périphériques. Leffectif des catégories apparait dans le coin supérieur gauche.

- Le vocabulaire commun à toutes les catégories représenté au centre.

- En l'espèce, sont retenus comme variables de contexte, les jalons suivants :

- Administration fiscale; Agents criminels; Banques; Blanchiment de capitaux; Comportement; Connaissances comptables ; Connaissances comptables ; Criminalité financière ; Criminel financier ; Criminels financiers ; État ; Évasion fiscale ; Expérience ; formation ; fraude fiscale ; Médias; Norme; Optimisation fiscale; organisation; Pouvoir; Régulation; Risque pénal ;

- La taille des mots est proportionnelle à leur fréquence. Dans les pavés catégories ne figurent que les mots surreprésentés. Les communs sont les plus fréquents parmi les mots communs à toutes les catégories.

$18 \mathrm{http} / /$ infos.lesphinx.eu/docquali/fr/presentation-de-sphinx-quali/glossaire.html\#varContexte (Consulté le 6 mai 2016). 